\title{
Differential binding capacity and internalisation of bacterial substrates as factors in growth rate of Acanthamoeba spp.
}

\author{
E. J. BOTTONE, A. A. PEREZ, R. E. GORDON* and M. N. QURESHI
}

Clinical Microbiology Laboratories and 'Department of Pathology, The Mount Sinai Hospital, One Gustave L. Levy

Place, New York, NY 10029, USA

\begin{abstract}
Summary. Acanthamoeba spp. are free-living predators that selectively feed on bacteria. Adherence of the bacterial food source to the trophozoite membrane is followed by internalisation and digestion. Through co-cultivation of $A$. castellanii and A. polyphaga, individually, with Xanthomonas maltophilia, Escherichia coli, Staphylococcus epidermidis and Pseudomonas aeruginosa (despite the amoebicidal properties of the latter organism), specificity with regard to the preferred bacterial substrate was judged. $X$. maltophilia and $P$. aeruginosa adhered almost immediately forming a multilayered mantle of bacilli around trophozoites of both species of amoebae. $E$. coli adhered to fewer trophozoites and in smaller numbers. $X$. maltophilia was readily internalised after co-cultivation for $8 \mathrm{~h}$, whereas $P$. aeruginosa, E. coli and $S$. epidermidis were not internalised even after co-cultivation for $24 \mathrm{~h}$. These data suggest that the suitability of a bacterial food source for the Acanthamoeba spp. studied is associated not only with the proclivity with which the bacterial species binds to the trophozoite surface, but also with the rate of its internalisation.
\end{abstract}

\section{Introduction}

Acanthamoeba spp. are free-living predators that selectively feed on diverse bacteria encountered in the environment. Germane to their feeding pattern is adherence of the bacterial food source to the trophozoite membrane with subsequent internalisation within phagosomes and digestion. Therefore, adherence and internalisation kinetics determine the suitability of the bacterial substrate which is in turn reflected in the growth rate of the acanthamoebae.

Our previous report ${ }^{1}$ showed that several waterborne environmental bacteria such as Xanthomonas maltophilia, Flavobacterium breve and Pseudomonas paucimobilis, which co-habitate with Acanthamoeba spp., supported growth of acanthamoebae better than the non-environmentally derived, but recommended, co-cultivating bacterial species Escherichia coli. ${ }^{2}$ In that study, counts of viable amoebae were made over a 96-h period, but little attention was paid to the definitive amoeba-bacterium interactions underscoring growth rate and selectivity of the bacterial substrate. In this study, through co-cultivation, light microscopy and electronmicroscopy, we examined the rate of adherence and internalisation of several gramnegative and gram-positive bacteria to $A$. castellanii and $A$. polyphaga.

\section{Materials and methods}

\section{Adherence and internalisation of bacteria}

A. castellanii (ATCC 30461) and A. polyphaga (ATCC 30010 ) axenically grown in proteose peptoneyeast extract-glucose medium ${ }^{2}$ were co-cultivated individually in sterile saline at $35^{\circ} \mathrm{C}$ with clinical isolates of $X$. maltophilia, E. coli, Staphylococcus epidermidis and, even though lethal to Acanthamoeba spp., P. aeruginosa. ${ }^{3,4}$ To individual saline suspensions containing $2 \times 10^{5}$ acanthamoebae $(70 \%$ trophozoites, $30 \%$ cysts $) / \mathrm{ml}, 1 \times 10^{5}$ cells of each bacterial species from bacterial growth harvested from sheep blood agar cultures incubated overnight at $35^{\circ} \mathrm{C}$ were added and gently mixed.

Immediately, and at 2-h intervals for $8 \mathrm{~h}$, and after $24 \mathrm{~h}$, a drop of the mixture was removed for examination by phase contrast microscopy and Gram's stain. Ten contiguous trophozoites were evaluated at each interval. The remaining suspension was processed for transmission electronmicroscopy (TEM). The cells for TEM were fixed in glutaraldehyde $3 \%$, buffered to pH 7.4 with sodium cacodylate, post-treated with osmium tetroxide $1 \%$, dehydrated in increasing concentrations of ethanol and embedded in EMbed 812. 


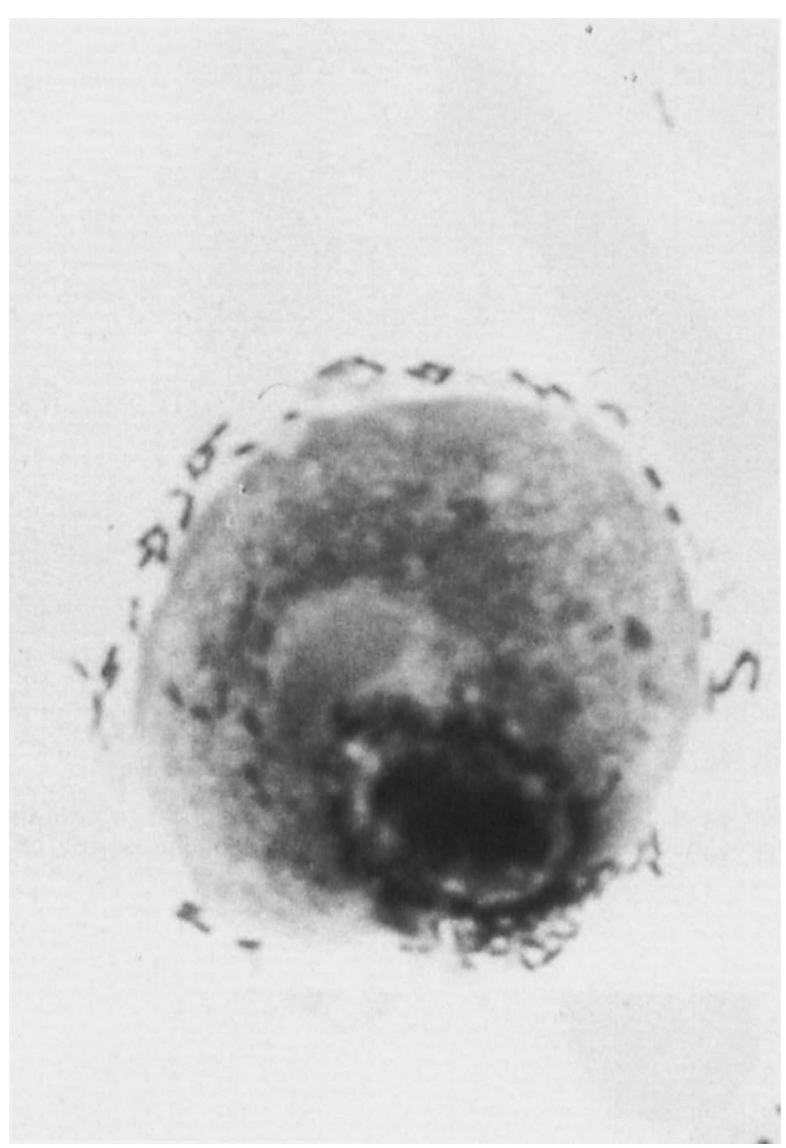

Fig. 1. Gram-stained smear showing linear adherence of $X$. maltophilia to $A$. polyphaga trophozoites after co-cultivation for $2 \mathrm{~min}$ in sterile saline $(\times 1000)$. (Similar results were noted for $A$. castellanii.)

\section{Results}

The $X$. maltophilia, $P$. aeruginosa and $E$. coli isolates adhered to trophozoites of both species of Acanthamoeba almost immediately; smears prepared immediately on mixing amoebae and bacteria showed bacilli in close proximity to the trophozoite membrane. However, counts of bacteria adhering to 10 trophozoites showed that the $X$. maltophilia and $P$. aeruginosa isolates were more adherent than $E$. coli. After co-cultivation for 2 min with $X$. maltophilia and $P$. aeruginosa, a mantle of bacilli encircled the trophozoite membrane and cyst forms (fig. 1). Initially, as noted with $X$. maltophilia, bacteria approached the trophozoite head-on and adhered in such a way that the distal end pointed into the medium. In fact, it appeared that the bacteria became entrapped in a surface matrix for an otherwise motile bacterium could be seen struggling to free itself.

By electronmicroscopy (fig. 2), the nature of the head-on articulation of $X$. maltophilia with the amoeba could be discerned better. At the attachment sites of the bacilli, the trophozoite plasmalemma was thicker and the trophozoite glycocalyx appeared to be lost. By way of contrast, the glycocalyx on the amoebic surface remained intact in the absence of full articulation by

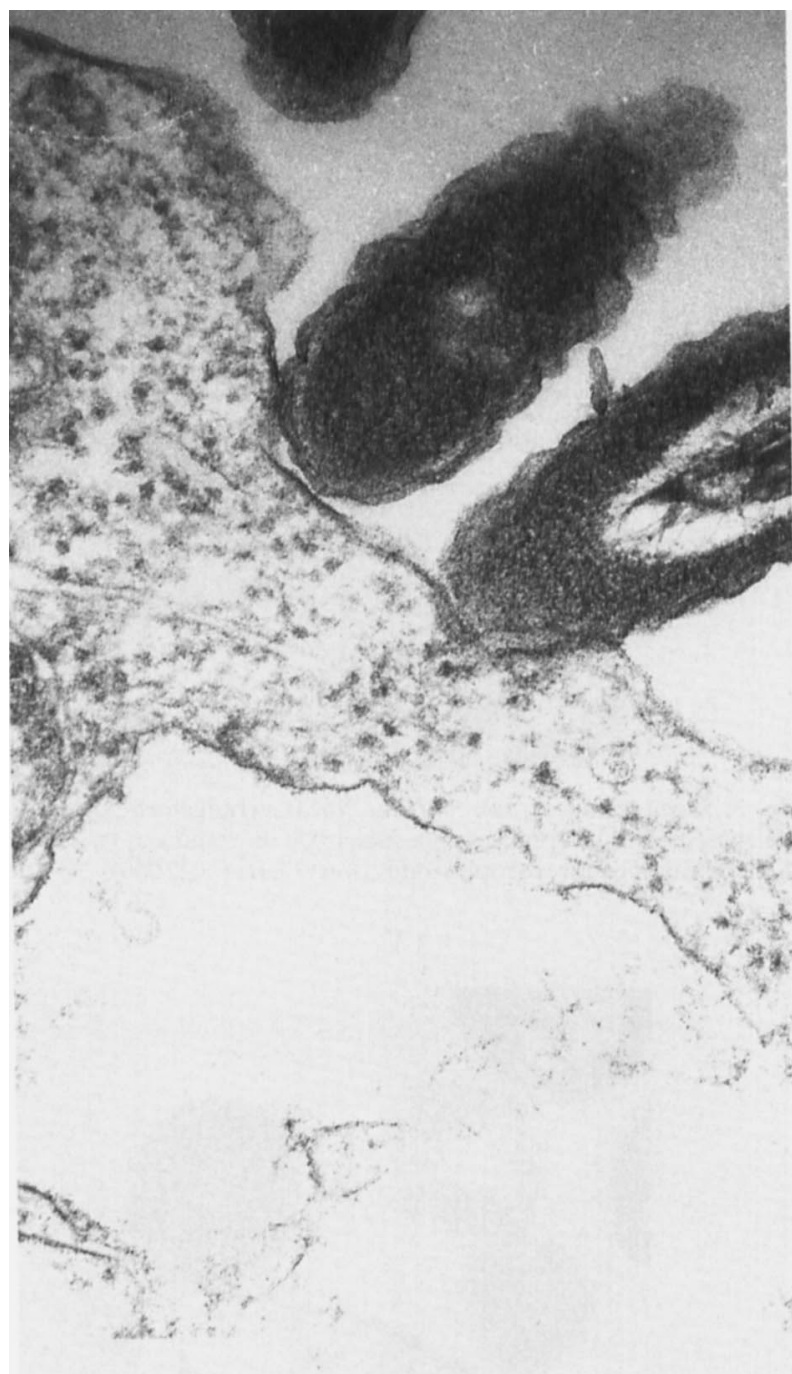

Fig. 2. Electronmicrograph showing two $X$. maltophilia cells articulating head-on with an $A$. polyphaga trophozoite at sites of plasmalemma thickening. A third $X$. maltophilia cell is seen approaching the trophozoite glycocalyx which is still intact $(\times 37000)$.

an approaching bacterial cell. In other fields, $X$. maltophilia was seen to attach to two distinct receptor sites on the same trophozoite and between three different trophozoites (fig. 3).

Subsequent to head-on articulation with the amoebic surface, these gram-negative bacteria assumed a linear position encircling the trophozoite membrane. In some instances, the mantle of adherent gram-negative bacteria delineated a clear zone distal to the cytoplasmic membrane of the trophozoite. This area may represent a potential glycocalyx which serves as the initial binding site leading to the gradual transposing of the attached bacteria to the amoebic membrane.

The adherence of the $S$. epidermidis isolate was similar to that observed for the gram-negative bacteria and included trophozoite plasmalemma thickening (fig. 4). Adherence seemed to be mediated through fibrils extending from the staphylococcal cell wall. 


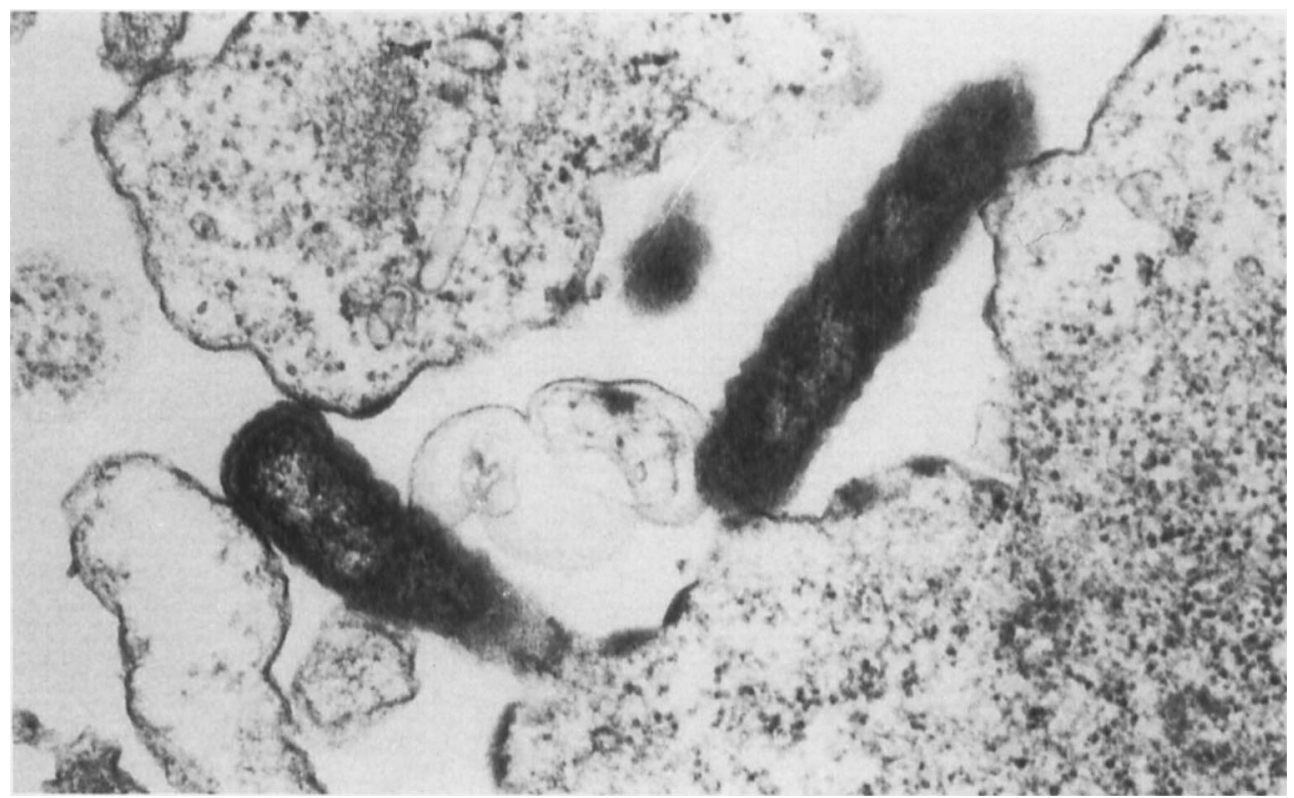

Fig. 3. Electronmicrograph showing linear arrangement of $X$. maltophilia on $A$. polyphaga trophozoite surface subsequent to head-on attachment. Note trophozoite plasmalemma thickening at two sites of bacterial attachment (upper right), and attachment of one bacterial cell to membranes of three trophozoites (lower left) $(\times 22000)$.

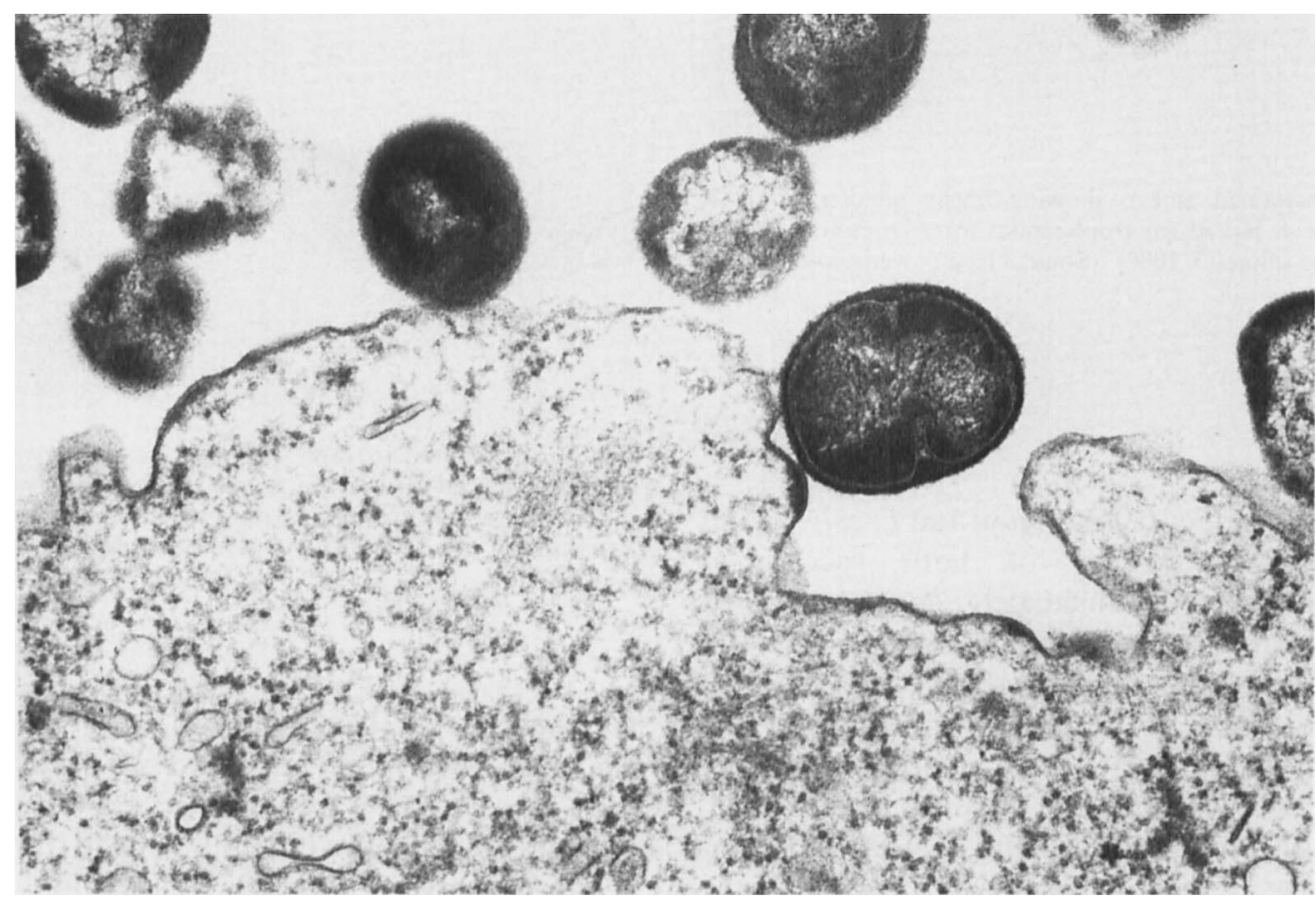

Fig. 4. Electronmicrograph showing adherence of a staphylococcal cell through fibrillar matrix to amoebic trophozoite membrane. Note trophozoite plasmalemma thickening at attachment sites $(\times 37000)$.

An interesting adherence phenomenon observed after co-cultivation for $24 \mathrm{~h}$ with $X$. maltophilia and, especially, with $P$. aeruginosa, was the focal clustering of multiple layers of bacteria to the trophozoite surface, and what appeared to be bacterial cell-to-cell adhesion forming a crown of attached organisms (fig. 5 ). Thus there was clear evidence for two definitive focal attachment sites on the amoebic surface. One comprised a two-three-cell layer and the other a mantle of adherent cells encompassing $75 \%$ of the trophozoite surface as if the bacteria were enveloped in a matrix secreted by the amoeba. However, by electronmicroscopy, there was an abundance of $P$. aeruginosa cells surrounding the trophozoite, but the absence of a clearly defined matrix to account for the focal adherence and the apparent cell-to-cell adherence.

Phagocytosis of adherent bacteria was most evident with $X$. maltophilia, occurring as early as within $8 \mathrm{~h}$ of co-cultivation. Electronmicroscopy revealed that 


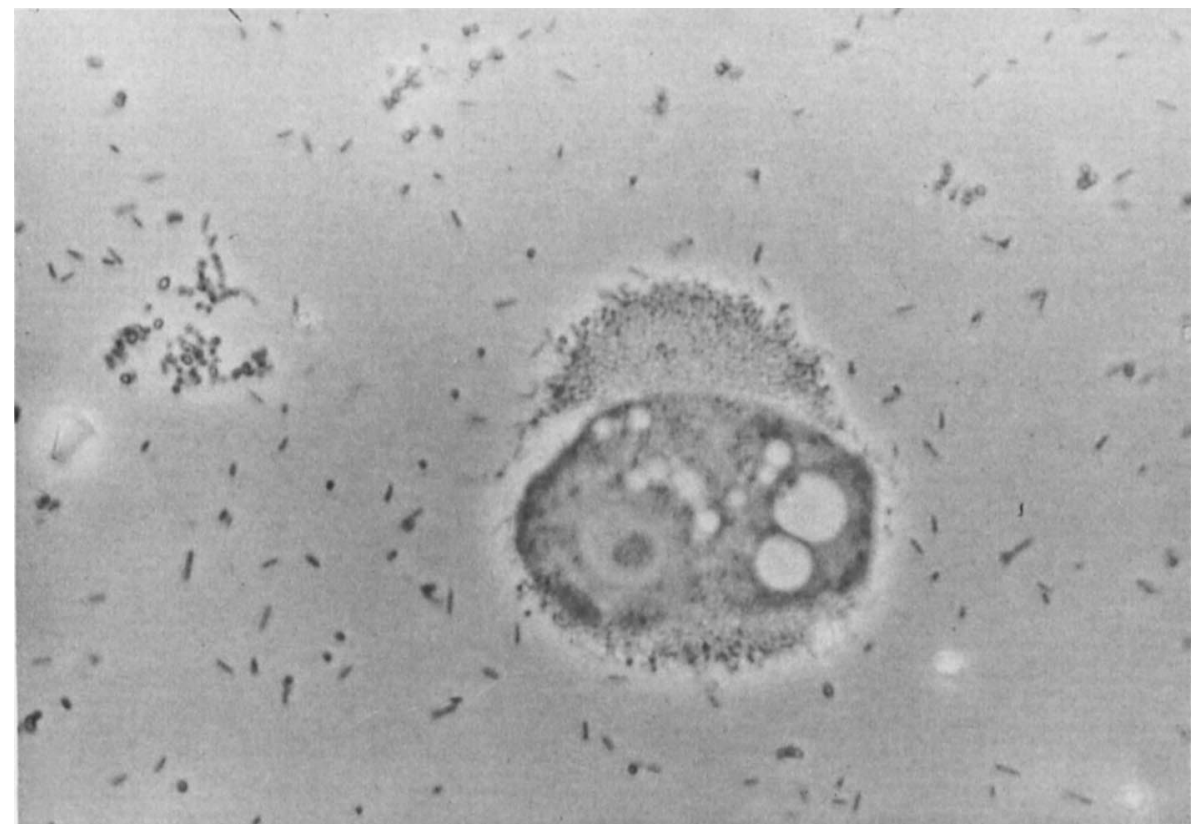

Fig. 5. Phase contrast photomicrograph of $A$. polyphaga trophozoite showing "capped" aggregation of $P$. aeruginosa cells after co-cultivation for $24 \mathrm{~h}$. Smaller cluster of adherent cells noted on lower aspect of trophozoite membrane $(\times 900)$. (Similar results were observed for $A$. castellanii).

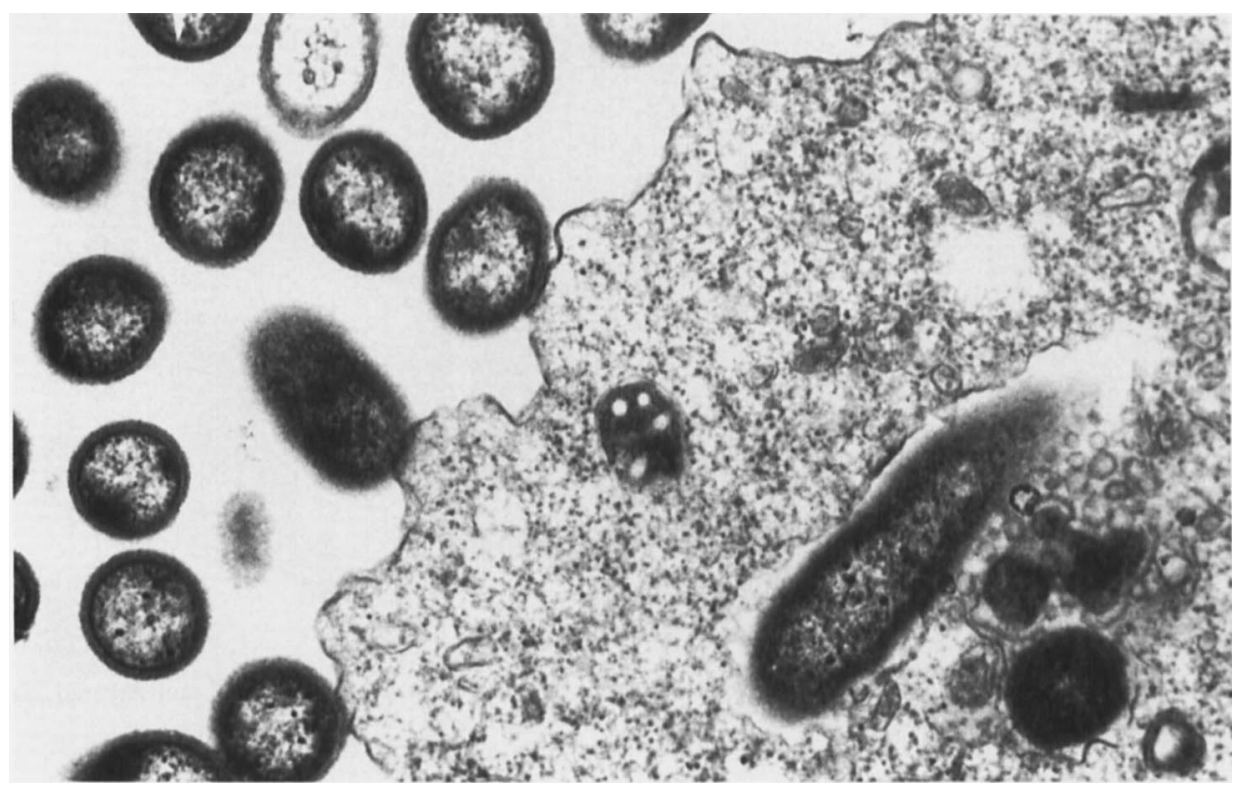

Fig. 6. Electronmicrograph taken after co-cultivation for $8 \mathrm{~h}$ of $X$. maltophilia with $A$. polyphaga showing adherent bacterial cells and several internalised within endocytic vacuoles $(\times 37000)$.

while there were still numerous $X$. maltophilia cells in linear adherence to the amoebic surface, many clearly discernible, internalised bacteria were observed within phagocytic vacuoles (fig. 6). After co-cultivation for $24 \mathrm{~h}$, some vacuoles contained up to 16 phagocytosed bacilli (fig. 7). By contrast, when E. coli was cocultivated with $A$. polyphaga and $A$. castellanii for $24 \mathrm{~h}$, despite the presence of numerous $E$. coli surrounding trophozoites, no internalisation of the bacteria could be seen. $P$. aeruginosa, which is lethal for both Acanthamoeba species, ${ }^{3}$ was found to adhere in large numbers to the amoebic surface, but only rarely was a bacterial cell internalised after co-cultivation for
$24 \mathrm{~h}$. This finding supports our earlier report ${ }^{3}$ that the pseudomonas amoebicidal activity ensues rapidly.

Interestingly, in gram-stained smears prepared after co-cultivation for $24 \mathrm{~h}$ with $S$. epidermidis (or $S$. aureus), cells were found layered around the entire trophozoite surface delineating its contour (fig. 8). However, internalisation was sparse, with only an occasional coccus found within a phagocytic vacuole. These observations were confirmed by electronmicroscopy. 


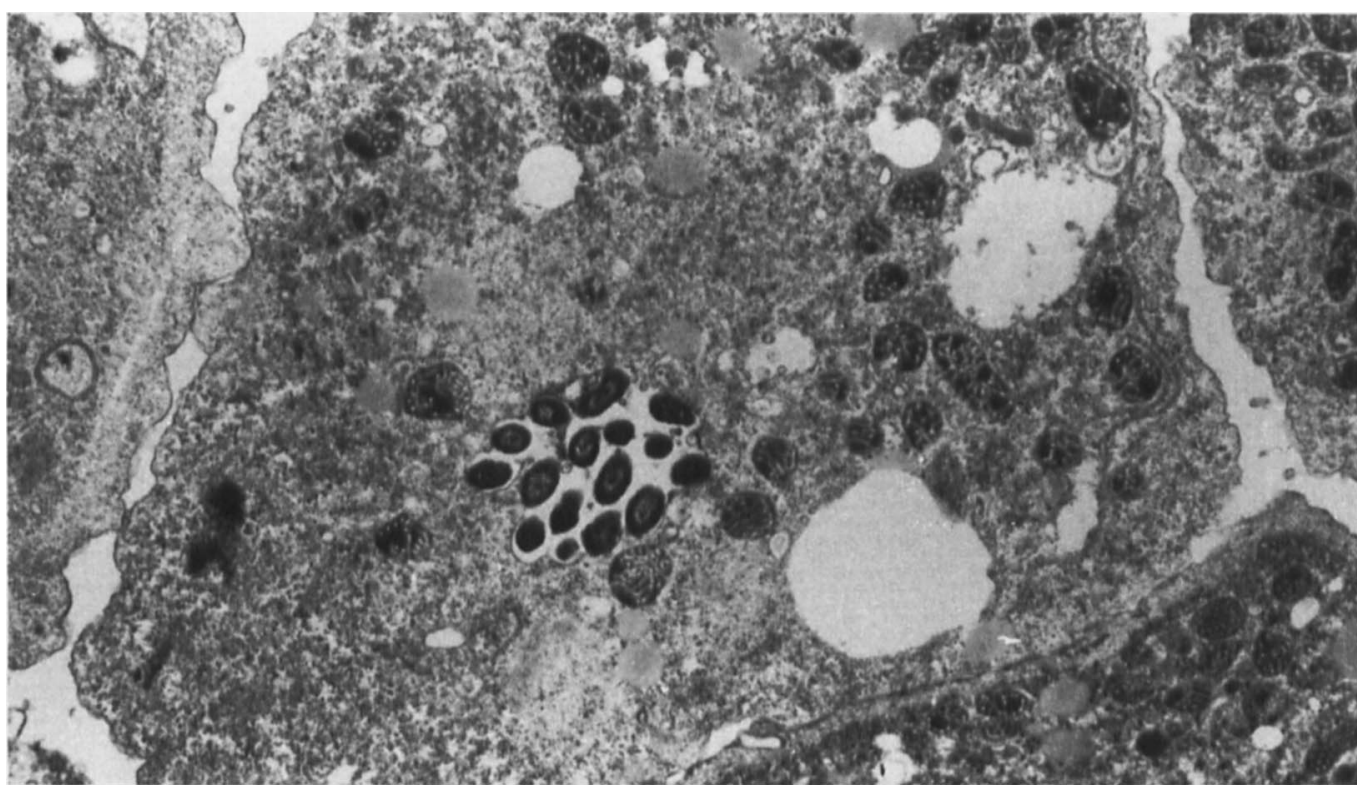

Fig. 7. Electronmicrograph showing $16 \mathrm{X}$. maltophilia cells within an endocytic vacuole in an $A$. polyphaga trophozoite $(\times 15000)$.

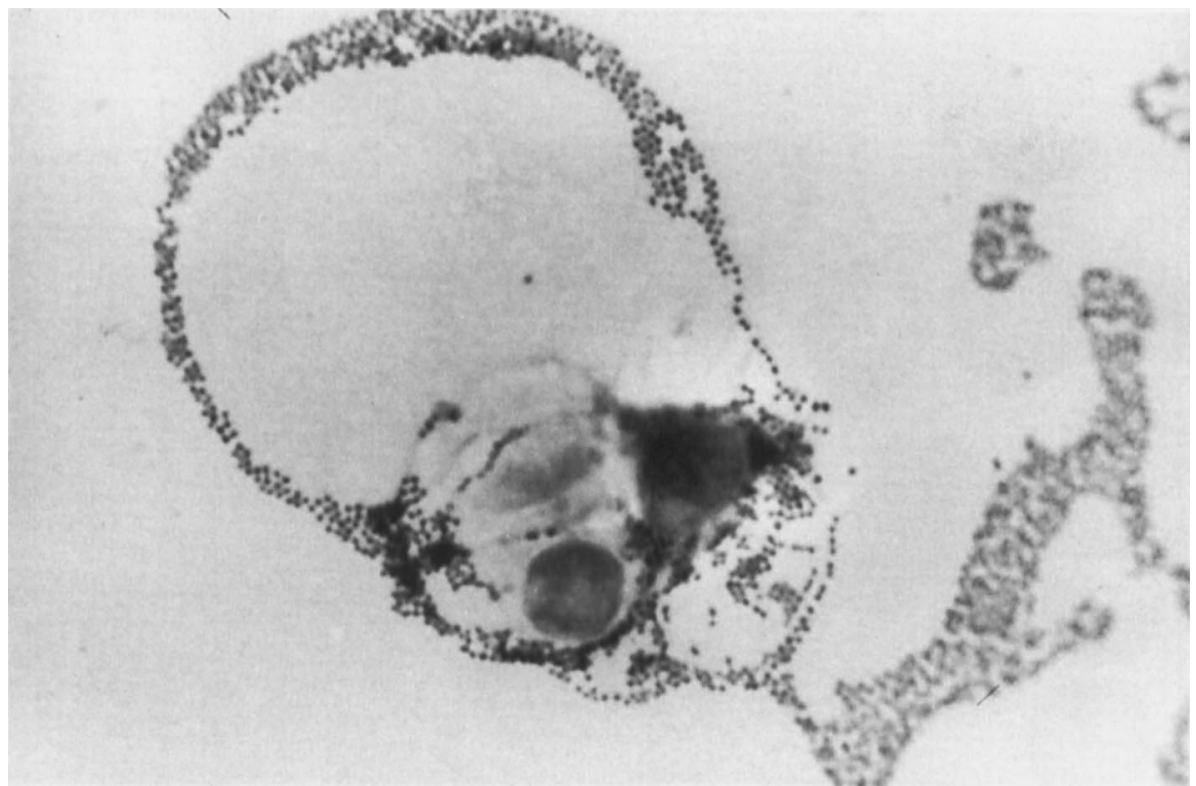

Fig. 8. Gram-stained smear of $S$. epidermidis co-cultivated with $A$. polyphaga for 24 h showing rim of multilayered staphylococcal cells surrounding trophozoite but sparse internalised cocci.

\section{Discussion}

Our studies have confirmed and advanced the earlier observations of Singh ${ }^{5}$ who showed a differential feeding tropism of free-living amoebae toward bacteria in their environment. Furthermore, we have shown that the suitability of a bacterial food source for Acanthamoeba spp. is intimately associated with the proclivity with which the bacteria bind to the trophozoite (and cyst) surface and the time course over which the bacterial substrate is internalised.

However, binding and internalisation are not equivalent steps in the growth cycle of Acanthamoeba spp. but vary with the nature of the bacteria. Thus, while many gram-negative and gram-positive bacteria bind to the amoebic surface, phagocytosis of bound bacteria, for an as yet unknown reason, is not equivalent for all species. Thus, whereas $X$. maltophilia was bound readily, and internalised within $8 \mathrm{~h}$ of cocultivation, $E$. coli, which also attached readily to the trophozoite surface, was not internalised even after cocultivation for $24 \mathrm{~h}$. These data augment our previous observations $^{1}$ of increased and earlier growth of $A$. castellanii and $A$. polyphaga in the presence of $X$. maltophilia over that with $E$. coli.

$P$. aeruginosa also bound readily to both the Acanthamoeba spp., forming a dense multilayered rim of adherent bacilli. From this repository, however, $P$. aeruginosa exerted an amoebicidal activity. ${ }^{3}$ In the present study, despite unbridled adherence, very few $P$. aeruginosa cells could be seen to be internalised after co-cultivation for $24 \mathrm{~h}$. As endocytosis by Acanthamoeba spp. is energy-dependent, ${ }^{6}$ failure to internalise what appears, by an adherence phenom- 
enon, to be a desirable food source, may actually be attributed to a pseudomonas-induced membrane lesion leading to the death of the amoebae. ${ }^{3}$

Acanthamoebae have also been shown to use certain gram-positive bacteria such as $S$. epidermidis as growth substrates. ${ }^{7}$ Our data showing marked adherence of $S$. epidermidis to trophozoites but delayed and sparse internalisation, as also noted with $E$. coli, confirm our previous observations on the equivalence of these two species as poor growth substrates for Acanthamoeba spp. Furthermore, our data provide a plausible explanation for the observations of Larkin and Easty, ${ }^{7}$ who showed that incubation for 6 days was required to determine the efficacy of $S$. epidermidis and $E$. coli as food sources for Acanthamoeba spp. inoculated on to lawns of these bacteria on agar.

During this study, we observed several phenomena relating to bacteria-amoebae adherence worthy of comment. By phase contrast microscopy of wet preparations, $X$. maltophilia cells were seen to be entrapped on the trophozoite surface and writhing in situ. This observation is similar to that reported by Preston and $\mathrm{King}^{8}$ who showed that $P$. fluorescens, a polar-flagellate bacterium analogous to $X$. maltophilia, attached to $A$. castellanii by its polar flagellum and, while anchored in this fashion, "produced a jostling monolayer around each amoeba". Our electronmicrographs clearly demonstrate $X$. maltophilia directly attached to and linking trophozoites. However, in several preparations, there was a clear space separating $X$. maltophilia cells from the amoebal surface which may have represented articulation through the flagellum which was either sheared off during preparation for electronmicroscopy or in a different section plane. Subsequent to attachment by flagella to the amoebic surface, the bacteria became apposed linearly on the trophozoite surface and, if determined suitable, endocytosed.

The second distinct phenomenon, occurring subsequent to the attachment of motile bacteria to trophozoites was the transposition of large numbers of the entrapped bacteria to a focal point(s) on the amoebic surface. It appeared that the amoebae were secreting a substance that ensnared and fixed multiple bacterial cells to their surface. In this fashion, phagocytosis of the entrapped food supply could proceed either gradually over time or as an entire unit. Compelling evidence for the secretion of an entrapping substance by the amoebae can be derived from a report by Weisman and Korn. ${ }^{6}$ Working with chemically inert latex beads, these investigators showed that, prior to phagocytosis, acanthamoebae first accumulated beads in closely packed aggregates on

\section{References}

1. Bottone EJ, Madayag RM, Qureshi MN. Acanthamoeba keratitis: synergy between amebic and bacterial cocontaminants in contact lens care systems as a prelude to infection. $J$ Clin Microbiol 1992; 30: 2447-2450.

2. Krogstad DJ, Visvesvara GS, Walls RW, Smith JW. Blood and tissue protozoa. In: Balows A, Hausler WJ, Herrmann KL, their membrane, then enveloped the beads with the plasma membrane and internalised them in a phagocytic vacuole.

Ray $^{9}$ also described a similar phenomenon for Hartmanella (Acanthamoeba) spp. in the presence of bacteria possessing a single polar flagellum. She showed that when $P$. aeruginosa and $P$. (Aeromonas) hydrophila impinged against the trophozoite surface, they stuck to and covered the trophozoite membrane. Subsequent bacterial impaction resulted in their accumulation on the amoebic surface and to each other until they were multilayered. The accumulated bacteria then began to focally coalesce or were "capped" on the amoebic surface. Ray further noted that pseudpodia arose occasionally from the amoebic surface on either side of the capped bacterial mass, converged toward each other, and endocytosed into a food vacuole a section of the bacterial mass abutting the cytoplasmic membrane. If undisturbed, the remaining bacterial mass remained fixed to the amoebic surface for continued digestion and new bacterial cells were added to the outermost layer. In our study, as well as that of Ray $^{9}$ and a 1902 report, ${ }^{10}$ capping was noted most frequently with polar flagellate bacteria such as Pseudomonas and Xanthomonas spp., less so with $E$. coli and not at all with $S$. aureus and $S$. epidermidis.

Our observations, through electronmicroscopy and light microscopy, of the feeding patterns of $A$. castellanii and $A$. polyphaga show that the suitability of a bacterial food source correlated directly with its ability to adhere to the trophozoite surface. In the case of polar flagellate environmental bacteria, such as Pseudomonas spp. (including $P$. aeruginosa) and $X$. maltophilia, attachment to available trophozoites in the co-cultivation system was uniform and immediate. Adhering bacteria could be focalised ("capped") and multilayered, suggesting the secretion by the amoebae of a "sticky" substance entrapping edible bacterial cells on their surface for internalisation, either sequentially or en masse.

Capping was not observed with $E$. coli, which is usually peritrichously flagellate, or the non-motile $S$. epidermidis, suggesting that this particular mode of feeding was absent for these bacteria. Thus, although these bacterial food sources bound readily to the amoebic surface, the delay in their internalisation in food vacuoles may be a function of the failure to be "capped" as a pre-requisite to phagocytosis. Interestingly, $\mathrm{Ray}^{9}$ showed that peritrichously flagellate bacterial cells formed haphazard clumps on the amoebic surface which may impede or delay phagocytosis.

Isenberg HD, Shadomy HJ (eds) Manual of clinical microbiology. 5th edn. Washington, D.C., American Society for Microbiology. 1991: 727-750.

3. Qureshi MN, Perez AA, Madayag RM, Bottone EJ. Inhibition of Acanthamoeba species by Pseudomonas aeruginosa: a rationale for their selective exclusion in corneal ulcers and contact lens care systems. J Clin Microbiol 1993; 31 : 1908-1910. 
4. Singh BN. The selection of bacterial food by soil amoebae, and the toxic effects of bacterial pigments and other products on soil protozoa. Br J Exp Pathol 1945; 26: 316-325.

5 . Singh BN. Selectivity in bacterial food by soil amoebae in pure mixed culture and in sterilized soil. Ann Appl Biol 1941; 28: 52-64.

6. Weisman RA, Korn ED. Phagocytosis of latex beads by Acanthamoeba. I. Biochemical properties. Biochemistry 1967; 6: 485-497.

7. Larkin DFP, Easty DL. External eye flora as a nutrient source for Acanthamoeba. Graefes Arch Clin Exp Ophthalmol $1990 ; 228$ : $458-460$.

8. Preston TM, King CA. Binding sites for bacterial flagella at the surface of the soil amoeba Acanthamoeba. J Gen Microbiol $1984 ; 130$ : 1449-1458.

9. Ray DL. Agglutination of bacteria: a feeding method in the soil ameba Hartmanella sp. J Exp Zool 1951; 118: 443-465.

10. Mouton $\mathrm{H}$. Recherches sur la digestion chez les amibes et sur leur diastase intracellulaire. Ann Inst Pasteur 1902; 16: 457-509. 